\title{
Quantum Dissipation due to the Interaction with Chaos
}

\author{
Doron Cohen ${ }^{1}$ and Tsampikos Kottos ${ }^{2}$ \\ 1 Department of Physics, Ben-Gurion University, Beer-Sheva 84105, Israel \\ ${ }^{2}$ Max-Planck-Institut für Strömungsforschung, Bunsenstraße 10, D-37073 Göttingen, Germany
}

\begin{abstract}
We discuss the possibility of having "quantum dissipation" due to the interaction with chaotic degrees of freedom. We define the conditions that should be satisfied in order to have a dissipative effect similar to the one due to an interaction with a (many body) bath. We also compare with the case where the environment is modeled by a random matrix model. In case of interaction with "chaos" we observe a regime where the relaxation process is non-universal, and reflects the underlaying semiclassical dynamics. As an example we consider a two level system (spin) that interacts with a two dimensional anharmonic oscillator.
\end{abstract}

The interaction of a system with its environment is a central theme in classical and quantum mechanics. The main effects that are associated with this interaction are "dissipation" (irreversible loss of energy) and "noise". The latter is due to "fluctuations" of the environmental degrees of freedom. On short time scales the main effect is the "decoherence" due to the noise. On long time scales the interplay of dissipation and noise leads to a state of thermal equilibrium.

The common modeling of the system-environment interaction is provided by a Hamiltonian $\mathcal{H}_{\text {total }}=\mathcal{H}_{0}+$ $\mathcal{H}(Q, P ; x)$, where $\mathcal{H}_{0}$ is the system Hamiltonian, $x$ is a system observable, and $\mathcal{H}(Q, P ; x)$ describes the environment including the interaction with the system. The simplest (and most popular) modeling of the environment is as a large collection of harmonic oscillators. This is known as the Caldeira-Leggett approach 1, 2]. Another approach is to use random matrix modeling of the environment 3, 4, 5]. However, in this paper we are interested in another possibility, where the interaction is with (few) chaotic degrees of freedom.

In what follows "interaction with chaos" means that the environment is the quantized version of a few degrees of freedom chaotic system. This should be contrasted with "interaction with bath" where the environment is modeled as a large collection of quantized harmonic oscillators. Let us regard the environment as a "black box" (one does not know what is there). The questions that we would like to address are: (1) How to characterize the bath in a way which does not assume a specific model. (2) Is it possible to distinguish "interaction with chaos" from "interaction with bath". As we explain below the second question is related to the notions of "thermodynamic limit" and "universality".

Common models for dissipation assume a "thermodynamic limit", which means interaction with infinitely many degrees of freedom. In this paper we inquire whether few chaotic degrees of freedom may have the same effect. This is a question of great practical importance. Future "quantum electronics" may consist of several interacting "quantum dots". One wonders how a coherent process in one part of the "circuit" is affected by the "noise" which is induced by the quantized chaotic motion of an electron in a nearby quantum dot. In other words: one would like to know whether it is possible to use in the nano-scale reality notions such as "dissipation" and "dephasing", that are traditionally associated with having an interaction with many degrees of freedom.

As a specific example we consider a two level system (spin) that interacts with a two-dimensional anharmonic oscillator. This would be the well known "Spin-Boson" model [1, 2] if the interaction were with a bath of harmonic oscillators. The motivation to deal with this model is well documented in the cited literature.

We also compare with the case where the interaction is with a random-matrix modeled environment. In the "quantum chaos" literature, and in mesoscopic physics, random matrix theory (RMT) is regarded as the "reference" case. Any deviation from RMT is called "nonuniversality", and has to do with the underlying semiclassical dynamics. In this paper we show that the notion of (non) universality can be extended into the studies of quantum dissipation.

The basic parameters that characterize any systemenvironment modeling are listed in Table 1 . We shall define these parameters in a way which is independent of the theoretical modeling of the bath. A common assumption is going to be that the mean level spacing is very small. For completeness of presentation, and for the purpose of defining what does it mean "very small", we keep $\Delta$ as an explicit free parameter (note [6]).

\begin{tabular}{|l|l|}
\hline parameter & \multicolumn{1}{|c|}{ significance } \\
\hline$\Delta \propto \hbar^{d}$ & environment mean level spacing \\
$\Delta_{b} \propto \hbar$ & environment bandwidth \\
$T$ & environment temperature \\
$d_{\mathrm{T}}$ & environment heat capacity \\
\hline$\epsilon$ & system energy \\
$A$ & system amplitude of motion \\
$V$ & system rate of motion \\
\hline$\Gamma$ & strength of coupling \\
\hline
\end{tabular}

Table 1: The various parameters that characterize a generic quantum dissipation problem. (See text for details). 
As a leading example we consider a two level system. For the system Hamiltonian we write $\mathcal{H}_{0}=(\hbar \Omega / 2) \boldsymbol{\sigma}_{1}$ where $\Omega$ is the Bloch frequency, and $\boldsymbol{\sigma}_{i=1,2,3}$ are the Pauli matrices. One can think of this Hamiltonian as describing a particle in a double well potential [1, 2]. Then it is natural to define its position as $x=v \sigma_{3}$, where $v$ is a constant. We assume that the interaction with the environment is via this "position" coordinate: $\mathcal{H}(Q, P ; x)=\frac{1}{2}\left(P_{1}^{2}+P_{2}^{2}+Q_{1}^{2}+Q_{2}^{2}\right)+(1+x) Q_{1}^{2} Q_{2}^{2}$. This environment can be interpreted as a particle moving in a 2 -dimensional anharmonic well $(2 \mathrm{DW})$. In the representation $|\nu, n\rangle$, which is determined by $\mathcal{H}(Q, P ; 0)$ and $\boldsymbol{\sigma}_{3}$, the Hamiltonian matrix takes the form

$$
H_{\text {total }}=\left[\begin{array}{cc}
\boldsymbol{E}+v \boldsymbol{B} & \hbar \Omega / 2 \\
\hbar \Omega / 2 & \boldsymbol{E}-v \boldsymbol{B}
\end{array}\right]
$$

where $\boldsymbol{E}=\operatorname{diag}\left\{E_{n}\right\}$ is a diagonal matrix that contains the energy levels of the environment, and $\boldsymbol{B}$ is a banded matrix (see $9 \mid$ ). The initial state of the total Hamiltonian $\Psi(t=0)$ is assumed to be factorized as $\varphi \otimes \psi$, where $\varphi$ is the initial state of the spin, and $\psi=\left|n_{0}\right\rangle$ is the initial state of the environment. It is implicit that we average over states with $E_{n_{0}} \sim E$ corresponding to a microcanonical preparation. As for the spin, we would like to consider the standard scenario where the initial state is a coherent superposition $|\varphi\rangle=(|\uparrow\rangle+|\downarrow\rangle) / \sqrt{2}$. The reduced probability matrix after time $t$ is

$$
\rho_{\nu, \nu^{\prime}}(t)=\sum_{n} \Psi_{\nu, n}(t)^{*} \Psi_{\nu^{\prime}, n}(t) \equiv \frac{1}{2}(1+\vec{M} \cdot \overrightarrow{\boldsymbol{\sigma}})_{\nu, \nu^{\prime}}
$$

where $\vec{M}=\left(M_{1}, M_{2}, M_{3}\right)$ is the polarization of the spin. It is most convenient to describe the state of the spin using $\vec{M}$. In particular we define $S(t)=\vec{M} \cdot \vec{M}$ as a measure for the purity of the spin state.

We turn to formulate the general case. The interaction of the system with the environment is assumed to be of the general form $\mathcal{H}_{\text {int }}=-x \mathcal{F}$ where $x$ and $\mathcal{F}$ are system and environmental observables respectively. (In the above example $x=v \boldsymbol{\sigma}_{3}$ and $\mathcal{F}=-Q_{1}^{2} Q_{2}^{2}$ ). In the absence of system-environment coupling we can characterize the fluctuations of the observable $\mathcal{F}(t)$ by a correlation function $C(\tau)$. (We are using here Heisenberg picture language). Its Fourier transform $\tilde{C}_{\mathrm{E}}(\omega)$ is known as the power spectrum of the fluctuations. The observable $\mathcal{F}$ has a matrix representation $\langle n|\mathcal{F}| m\rangle=-\boldsymbol{B}_{n m}$ where we use the basis which is determined by $\mathcal{H}$. The fluctuations are related to the bandprofile of this matrix:

$$
\begin{aligned}
\tilde{C}_{\mathrm{E}}(\omega) & =\left[\sum_{m}\left|\boldsymbol{B}_{n m}\right|^{2} 2 \pi \delta\left(\omega-\frac{E_{m}-E_{n}}{\hbar}\right)\right]_{E_{n} \sim E} \\
& \equiv 2 \pi \sigma^{2} \delta(\omega)+\frac{2 \pi \hbar \sigma^{2}}{\Delta} R\left(\frac{\hbar \omega}{\Delta}\right) G\left(\frac{\hbar \omega}{\Delta_{b}}\right)
\end{aligned}
$$

In the first expression there is an implicit microcanonical averaging over the states $E_{n} \sim E$. In the second expression $\sigma^{2}$ is the average value $\overline{\left|\boldsymbol{B}_{n m}\right|^{2}}$, taken over the neardiagonal matrix elements. The lower cutoff function $R()$ depends on the level spacing statistics $[7]$ : It is $R() \approx 1$ for $\omega>\Delta / \hbar$. The mean level spacing $\Delta$ is proportional to $\hbar^{d}$, where $d$ is the the number of environmental degrees of freedom. Any chaotic motion is characterized by a finite correlation time $\tau_{c}$. Therefore $\tilde{C}_{\mathrm{E}}(\omega)$ has a a cutoff frequency $\omega_{c}=2 \pi / \tau_{c}$. This implies (via Eq.(3)) that $\boldsymbol{B}_{n m}$ is a banded matrix with a bandwidth $\Delta_{b}=\hbar \omega_{c}$. The envelope function $G()$, with $G(0) \equiv 1$, describes the bandprofile.

For sake of comparison we refer to the Spin-Boson model. The distribution of the bath oscillators is described 1, 2] by a spectral function $J(\omega)$, leading to $\tilde{C}_{\mathrm{E}}(\omega)=2 \hbar J(\omega) /\left(1-\mathrm{e}^{-\beta \hbar \omega}\right)$, where $\beta$ is the reciprocal temperature of the bath. The "ohmic" assumption of having "white noise" $(C(\tau)$ with short correlation time $\left.\tau_{c}\right)$ for high temperatures is imposed by construction, by setting $J(\omega) \propto \omega G\left(\omega / \omega_{c}\right)$. This corresponds to a strong chaos assumption. However, it should be realized that even if we "cook" a harmonic bath that has the same $\tilde{C}_{\mathrm{E}}(\omega)$ as that of a chaotic environment, still there is a major difference: In spite of having the same bandprofile, the $\boldsymbol{B}_{n m}$ matrix of a harmonic bath is very sparse: only states that differ by "one photon" excitation of a single oscillator are coupled (hence the differences $E_{m}-E_{n}$ are the frequencies of the harmonic oscillators).

In what follows we would like to define the notion of temperature $(T)$ without assuming a specific modeling. There are three features of the environment that has to do with this notion: (i) The growing density of states; (ii) The growing fluctuations intensity; (iii) The asymmetry of $\tilde{C}_{\mathrm{E}}(\omega)$ with respect to $\omega$. Let us regard the system as a "thermometer". The equilibrium is determined by the microcanonical temperature $T=\left(\partial_{E} \ln (1 / \Delta)\right)^{-1}$. For $d=2$ environment with constant density of states we would get $T=\infty$. [We consider this hypothetical case for argumentation purpose. This would be indeed the case if the environment were modeled as a billiard. In a later paragraph we discuss the model of Eq.(1) for which $T$ is finite]. In case of a two level system, having $T=\infty$ implies an equal probability for the two energy states. Now we can take (instead of a two level system) a particle with one degree of freedom as a "thermometer". Since we already know that the "temperature" is (say) $T=\infty$, we may deduce that the friction coefficient, as determined by the fluctuation-dissipation theorem is $\eta=\nu /(2 T)=0$, where $\nu=\tilde{C}_{\mathrm{E}}(\omega \sim 0)$ is the intensity of the fluctuations. This conclusion is wrong. In fact the friction coefficient is $\eta=\frac{1}{2} \Delta \times \partial_{E}(\nu / \Delta)$, as discussed in [8] (and references therein). Thus we can define an effective temperature, which is related to the thermalization process:

$$
T_{\text {eff }} \equiv \frac{\nu}{2 \eta}=\left(\frac{\partial}{\partial E} \ln \left(\frac{1}{\Delta} \tilde{C}_{\mathrm{E}}(\omega \sim 0)\right)\right)^{-1}
$$

In generic circumstances the distinction between $T_{\text {eff }}$ and $T$ is not so dramatic. In fact, some further inspection into Eq.(3) reveals that generically $T_{\text {eff }}=T / 2$. The above subtlety does not apply to a thermal preparation. As- 
suming a reciprocal temperature $\beta$, the friction coefficient (as obtained by canonical averaging over the above cited microcanonical result) is $\eta=\frac{1}{2} \beta \nu$. Therefore we get $T_{\text {eff }}=1 / \beta$ as expected.

Having defined $T$, we can make a quantum mechanical distinction between high and low temperature regimes. This is related to the asymmetry of $\tilde{C}_{\mathrm{E}}(\omega)$ with respect to $\omega$. Some inspection into Eq.(3) reveals that a finite temperature implies that the band profile acquires a factor $\exp \left(\omega /\left(2 T_{\text {eff }}\right)\right)$, which is consistent with the Spin-Boson modeling (see a previous paragraph). "High temperature" means that for the physically relevant frequencies $\hbar \omega / T \ll 1$. A sufficient condition is $T \gg \Delta_{b}$. In the latter case $\tilde{C}_{\mathrm{E}}(\omega)$ can be treated as a symmetric function with respect to $\omega \mapsto-\omega$, and therefore it can be interpreted as the power spectrum of a classical noise.

The issue of thermodynamic limit is related to the heat capacity of the environment. This is $d_{\mathrm{T}}=(\partial T / \partial E)^{-1} \sim$ $d$, where $d$ is its number of environmental degrees of freedom. The energy that the system can exchange with the environment is denoted by $\epsilon$. If we want to assume a stable temperature $T$, the heat capacity of the environment should be large enough, so that energy exchange between the system and the environment does not have a big effect. This leads to the condition $\epsilon \ll d_{\mathrm{T}} \times T$. For a generic few degrees of freedom system $\epsilon \sim d_{0} \times T$, where $d_{0}$ is the number of system degrees of freedom. This leads to $d_{0} \ll d_{\mathrm{T}}$. In case of a two level system the condition is much easier. Namely, we have $\epsilon \sim \hbar \Omega$, and therefore we get the "easy" condition $\hbar \Omega \ll d_{\mathrm{T}} \times T$.

Assuming the typical circumstances of an oscillating system, the time variation of the system observable $x(t)$ is characterized by an amplitude $|x| \sim A$, and by a rate of change $|\dot{x}| \sim V$. This specification of the system dynamics is essential in order to define a dimensionless parameter that characterizes the system-bath interaction:

$$
\frac{\Gamma}{\Delta}=\operatorname{minimum}\left(\frac{2 \pi \sigma^{2}}{\Delta^{2}} A^{2},\left(\frac{\hbar \sigma}{\Delta^{2}} V\right)^{2 / 3}\right)
$$

Loosely speaking this parameter indicates how many environmental energy levels are mixed non-perturbatively due to the interaction with the system. The $A$ dependence of $\Gamma$ is the consequence of the well known theory by Wigner: It is the number of levels which are mixed by the perturbation in $\mathcal{H} \mapsto E_{n} \delta_{n m}+x \boldsymbol{B}_{n m}$ with $|x| \sim A$. If the perturbation is slow (small $\dot{x}$ ) this $A$ based estimate becomes non-relevant. For a proper analysis [8] one should switch to the adiabatic ( $x$ dependent) basis, leading to $\mathcal{H} \mapsto E_{n} \delta_{n m}+\dot{x}\left(i \hbar \boldsymbol{B}_{n m} /\left(E_{n}-E_{m}\right)\right)$ with $|\dot{x}| \sim V$. The $V$ based estimate for $\Gamma$ follows from the latter representation. Thus one realizes that for slow rate of $x$ variation there is a crossover form a $A$-determined to a $V$-determined mixing.

The rest of this paper is aimed in clarifying the significance of the parameter $\Gamma$. Some results of the simulations with the 2DW model Eq.(11) are presented in Fig.1. The energy of the environment was in the range $2.8<E<3.2$ where the classical dynamics is predominantly chaotic. The classical correlation time is $\tau_{c} \sim 1$.
The simulations are done with $\hbar=0.03$. This means that the bandwidth is $\Delta_{b} \sim 0.2$. This should be contrasted with the mean level spacing $\Delta \sim 0.004$. The temperature in the specified energy range is $T \sim 1.3$, and the heat capacity is $d_{\mathrm{T}} \sim 2.4$. The amplitude of the motion is $A=v$, while the rate of the motion is formally $V=\infty$. The latter should be understood in the path-integral context (Eq. (7)), where $x(t)$ makes "jumps" between the two sites $(x= \pm v)$. Hence $\Gamma \propto(v / \hbar)^{2}$. One can easily verify that the Kondo parameter [2] is $\alpha=(1 / 16 \pi) \Gamma / T$. In our simulations $\alpha \ll 1$.

In the lower panels of Fig.1 we present the corresponding results of simulations with a RMT model where the induced fluctuations have exactly the same power spectrum as in the 2DW model. The RMT model has been obtained by taking the Hamiltonian Eq.(1) with a randomized $\boldsymbol{B}$. We simply randomized the signs of the offdiagonal elements. This procedure destroys all the correlations between the elements, but does not affect the bandprofile (which is implied by Eq.(31)). Two observations should be made immediately: (i) A few degrees of freedom chaos indeed provides a dissipative effect, as in the case of a many degrees of freedom bath. (ii) The effect of interaction with "chaos" can be distinguished from the case of a random-matrix modeled environment. The latter claim is based on the observation that in the regime $v>0.16$ there is a two orders of magnitude difference between the corresponding curves. Also the scaling of curves with respect to $v$ is "broken" (upper panel): The sensitivity to $v$ is much smaller than implied by the overcompensating scaling of the time axis.

As explained in the introduction the deviation from RMT is regarded as "non-universality" and its understanding requires a proper definition of the classical limit. This should not be mistaken as a synonym for "high temperatures". The confusing, and possibly meaningless procedure to define this limit is by taking $\hbar \rightarrow 0$. We argue below that the meaningful definition of a "semiclassical regime" is by considering the nature of the dynamics of the environment. This leads to the condition $\Gamma \gg \Delta_{b}$. In order to explain this condition we adopt the Feynman-Vernon picture of the dissipation process. Within this picture the propagator of the reduced probability matrix is written as a path integral:

$$
K\left(\nu, \nu^{\prime} \mid \nu_{0}, \nu_{0}^{\prime}\right)=\sum_{x_{A}, x_{B}} F\left[x_{A}, x_{B}\right] \mathrm{e}^{i\left(\mathcal{A}\left[x_{A}\right]-\mathcal{A}\left[x_{B}\right]\right)}
$$

The summation is over pairs of system trajectories (with weight factors absorbed into the definition of the integration measure). The action $\mathcal{A}[x]$ is defined as the phase which is accumulated along a given trajectory. We note that for a spin the trajectory is piecewise constant $(x= \pm v)$. The influence functional is defined as $F\left[x_{A}, x_{B}\right]=\left\langle\psi\left|U\left[x_{B}\right]^{-1} U\left[x_{A}\right]\right| \psi\right\rangle$ where the expectation value is taken for the initial preparation of the environment (it is typically a mixture of many states implying that an appropriate average should be taken over $\psi$ ). The environmental evolution opera- 
tor in case of the models that we consider is $U[x]=$ $\mathcal{T} \exp \left(-(i / \hbar) \int_{0}^{t}\left(\boldsymbol{E}+x\left(t^{\prime}\right) \boldsymbol{B}\right) d t^{\prime}\right)$. Thus, in a semiclassical framework the problem of "quantum dissipation" reduces to that of analyzing "driven degrees of freedom". It has been realized 10] that the driven dynamics becomes non-perturbative if $\Gamma>\Delta_{b}$. Below we discuss the implication of this observation.

The purity $S(t)$ is related to the dephasing factor $\left|F\left[x_{A}, x_{B}\right]\right|$. [For $\Omega=0$, the Hamiltonian Eq. (1) becomes block diagonal, leading to $S(t)=\left|F\left[x_{A}, x_{B}\right]\right|^{2}$, with $x_{A}=v$ and $x_{B}=-v$ ]. If the fluctuations are regarded as "noise", one obtains the standard expression $\left|F\left[x_{A}, x_{B}\right]\right|=\mathrm{e}^{-\frac{1}{2 \hbar^{2}} \int_{0}^{t} \int_{0}^{t} C\left(t^{\prime}-t^{\prime \prime}\right)\left(x_{B}\left(t^{\prime}\right)-x_{A}\left(t^{\prime \prime}\right)\right)^{2} d t^{\prime} d t^{\prime \prime}}$. This expression implies a short time Gaussian decay $S(t)=\exp \left(-4 C(0)(v t / \hbar)^{2}\right)$, which evolves into a long time Gaussian decay $S(t)=\exp \left(-(\sigma v t / \hbar)^{2}\right)$ in the regime $\Gamma<\Delta$. If the envelope $G()$ of the bandprofile were smooth, then we would expect, in the regime $\Delta<\Gamma<\Delta_{b}$, an intermediate stage of exponential decay $S(t)=\exp (-2 \gamma t)$ with $\gamma=2(v / \hbar)^{2} \tilde{C}_{\mathrm{E}}(\Omega) \sim \Gamma / \hbar$. In case of our numerical example the bandprofile has a structure [9], and hence $C(\tau)$ has oscillations that show up in the simulations.

Can we trust the standard expression for $\left|F\left[x_{A}, x_{B}\right]\right|$ if we have a dynamical environment rather than a noise source [with the same $C(\tau)$ ]? It is not difficult to observe that the standard expression can be derived from Fermigolden-rule (FGR) considerations. In case of Harmonic bath the FGR treatment is valid, and this expression, with $C(\tau)$ replaced by its symmetrized version, is exact. But for interaction with "chaos" we have claimed above that non-universality should show up in the nonperturbative regime $\left(\Gamma>\Delta_{b}\right)$. Our numerics confirm this prediction: In the non-perturbative regime $\left(\Gamma>\Delta_{b}\right)$ we find a premature $\left(t<\tau_{c}\right)$ crossover from the expected short time Gaussian decay, to a non-universal behavior which is not captured by the standard formula. In the 2DW case the system has a classical limit, and therefore the nonperturbative decay of $S(t)$ is slowed down (compared with RMT) because it is limited by the classical dynamics. As observed (note the inset) the decay becomes much less sensitive to $v$.

The above discussed non-universality can be regarded as the manifestation of "semiclassical" correlations between the off-diagonal matrix elements of $\boldsymbol{B}_{n m}$. These are not reflected in $\tilde{C}_{\mathrm{E}}(\omega)$. In case of the Spin-Boson model the $\boldsymbol{B}_{n m}$ matrix is sparse, which implies (in the limit of infinite bath) that off-diagonal correlations can be neglected. In case of a random matrix modeled environment, absence of correlations is guaranteed by construction for $x=0$, which implies (assuming no sparsity) lack of "invariance" with respect to $x$ [11]. Accordingly we have three classes of models that become distinct in the non-perturbative regime.

In conclusion, we have discussed the consequences of having four energy scales $\left(\Delta, \Delta_{b}, T, \Gamma\right)$ in any generic problem of "quantum dissipation". The strength of the interaction is characterized by $\Gamma$. A universal "quantum dissipation" behavior requires a separation of energy scales $\Delta \ll \Gamma \ll \Delta_{b}$. Non-perturbative breakdown of this universality due to the underlaying semiclassical dynamics is found if $\Gamma>\Delta_{b}$.

We thank M. Esposito, P. Gaspard, L. Pastur, and V. Falco for stimulating discussions. This work was supported by a Grant from the GIF, the German-Israeli Foundation for Scientific Research and Development, and by the Israel Science Foundation (grant No.11/02).

[1] Quantum Dissipative Systems, U. Weiss, World Scientific, Singapore (1999).

[2] A.J. Leggett et al, Rev. Mod. Phys. 59, 1 (1987).

[3] J. Lebowitz and L. Pastur, preprint (2002).

[4] M. Esposito and P. Gaspard, Phys. Rev. E 68, 066113 (2003); Phys. Rev. E 68, 066112 (2003).

[5] P. Pereyra, J. Stat. Phys. 65, 773 (1991). P.A. Mello, P. Pereyra and N. Kumar, J. Stat. Phys. 51, 77 (1988).

[6] From here on we ignore the effect of recurrences that happen after an extremely large time.

[7] O.M. Auslaender and S. Fishman, J. Phys. A 33, 1957 (2000); Phys. Rev. Lett. 84, 1886 (2000).

[8] D. Cohen, Annals of Physics 283, 175 (2000).

[9] D. Cohen and T. Kottos, Phys. Rev. E 63, 36203 (2001).

[10] D. Cohen, Phys. Rev. Lett. 82, 4951 (1999). D. Cohen and T. Kottos, Phys. Rev. Lett. 85, 4839 (2000).

[11] Attempts to have an $x$ invariant random matrix model, [such as in P. A. Bulgac et al, PRE 58, 196 (1998)], make the model "perturbative by construction" [10]. 


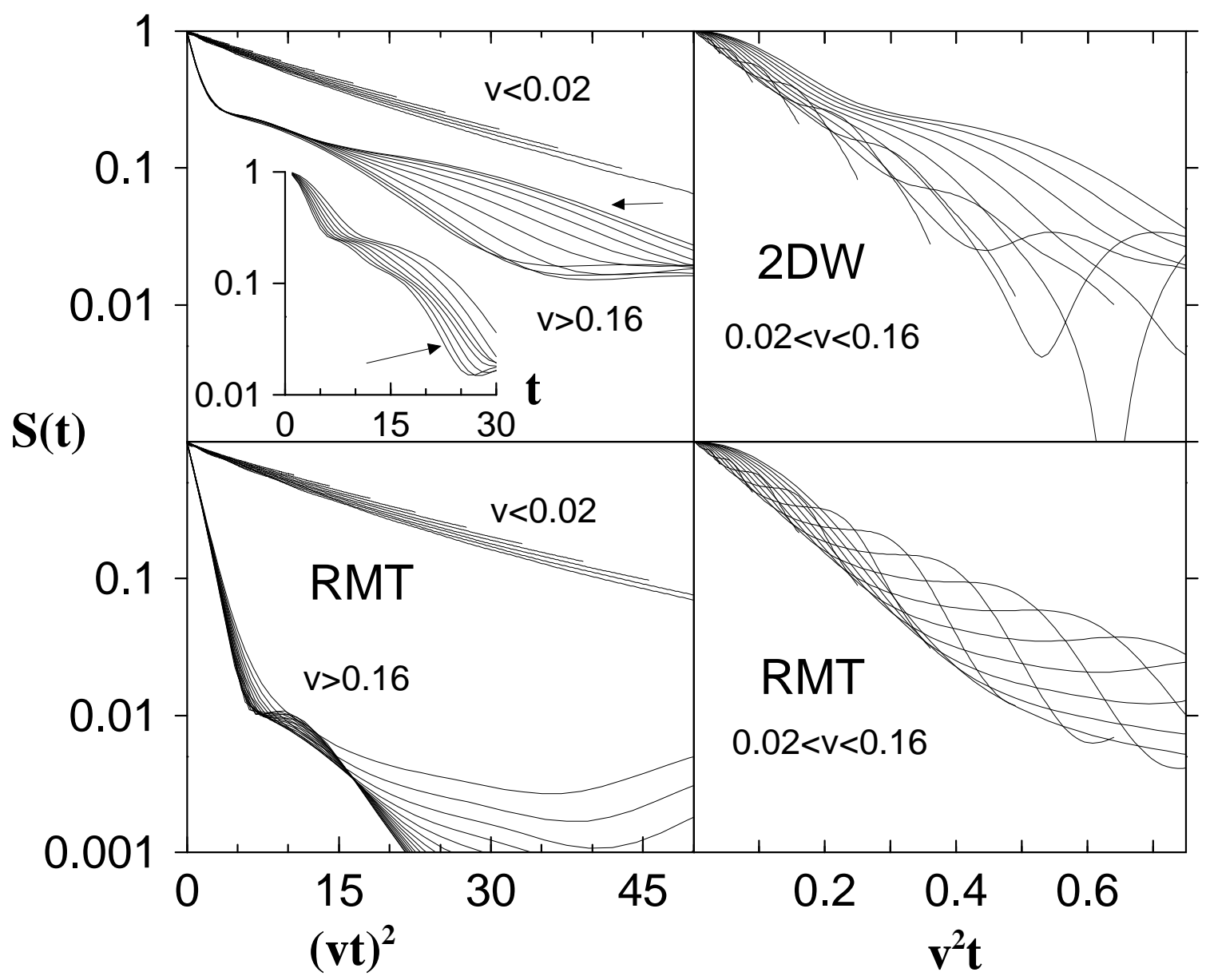

FIG.1: The decay of $S(t)$ in case of interaction with chaos (upper panels) and in case of interaction with a "randomized" environment that has exactly the same fluctuations (lower panels). The selected values of the coupling parameter are in the range $10^{-4}<v<0.3$. The left panels are for the $\Gamma<\Delta$ regime $(v<0.02)$ and for the $\Gamma>\Delta_{b}$ nonperturbative regime $(v>0.16)$. The inset is for some of the $v>0.16$ curves without scaling. The arrows point on the largest $v$ value. The right panels are for the $\Delta<\Gamma<\Delta_{b}$ regime $(0.02<v<0.16)$. The scaling methods of the time axis are implied by the analysis of $S(t)$, which should hold if there is a universal behavior which is determined by $C(\tau)$ alone (see text). 